\title{
Symplocos nicolsonii, (Symplocaceae) a new species from the Southern Western Ghats, India.
}

Jagadeesan R. ${ }^{1}$, Gangaprasad A. ${ }^{1}$, Sam P. Mathew ${ }^{2 *}$, Santhosh Kumar E. S. ${ }^{2}$, J. Stephan ${ }^{2}$

${ }^{1}$ Department of Botany, University of Kerala, Kariavattom P.O., Thiruvananthapuram, Kerala, India. 2Jawaharlal Nehru Tropical Botanic Garden and Research Institute, Palode, Karimankode P.O., Thiruvananthapuram, Kerala, India.

Received: July 6, 2016; Accepted: July 27, 2016.

Abstract: Symplocos nicolsonii Jagadeesan et al. closely allied to Symplocos lancifolia Siebold. \& Zucc., from the Southern Western Ghats is described and illustrated here as a new species.

Key words: Symplocos nicolsonii; New species; Symplocaceae; Southern Western Ghats.

\section{Introduction}

The genus Symplocos Jacq. comprises about 250 300 species distributed from South to Southeast Asia, America, Australia and East Indies (Brand, 1901; Nooteboom, 1975; Stahl, 1995). The official website of Kew Herbarium (The Plant List, A working list of plant species) enumerated 219 accepted taxa beneath the genus Symplocos Jacq. (http:/ / www.theplantlist.org/tpl1.1/search?q=Sy mplocos: accessed on 28/06/2016). It consists of 32 taxa in India, mostly included under the section Lodhra G. Don, characterized with an articulation found between the pedicel and calyx tube, petals connate at base, terete staminal filaments and 3 celled endocarp (rarely 1-celled) (Fritsch et al., 2008). Nair et al., 2014 enumerated 14 species and 11 subspecies for the genus Symplocos from the Western Ghats.

During the investigations on endemic flora of the Southern Western Ghats, the authors have come across an interesting Symplocos from the Mathikettan Shola National Park of the Idukki District in Kerala State. On critical taxonomic investigations and perusal of literature revealed that the taxon is morphologically distinct with other known species of the genus. On scrutiny, the newly collected plant specimen has been recognized under section Lodhra G. Don and allied to Symplocos lancifolia Siebold. \& Zucc. The new taxon named as Symplocos nicolsonii is described herewith with photographs.

Symplocos nicolsonii R. Jagadeesan, A. Gangaprasad, S. P. Mathew, E. S. S. Kumar et J. Stephan sp. nov. (Fig.1.)

Diagnosis: Symplocos nicolsonii sp nov. principally differs from Symplocos lancifolia in having ellipticobovate brittle leaves, pubescent calyx tube, lacerate calyx, pubescent disc and grooved

\section{${ }^{*}$ Corresponding Author:}

Dr. Sam P. Mathew,

Scientist E1,

Jawaharlal Nehru Tropical Botanic Garden and Research Institute

Karimoncod PO, Palode, Trivandrum 695 562,

Kerala State, India.

E-mail: sampmatthew@gmail.com endocarp. Detailed characterization is tabulated below (Table 1).

Type: India, Kerala State, Idukki District, Mathikettan Shola National Park, \pm 2200 m, 12. 03. 2016, R. Jagadeesan 8074, (Holotype: CAL; Isotype, TBGT, KUBH)

Paratype: India: Kerala, Idukki District, Mathikettan Shola, 2300 m, 21.04.2016 R. Jagadeesan 9026 (KUBH).

Evergreen trees up to $15 \mathrm{~m}$ tall; bark rough, brownish grey; blaze pale green-yellow; young branchlets pubescent with brownish hairs, stout with prominent leaf scars on maturity, Leaves simple, alternate, exstipulate, $2-5.5 \times 1-3.5 \mathrm{~cm}$, elliptic-obovate, acute to acuminate at apex, cuneate at base, glandular, serrated or rarely revolute at margin, brittle, midrib impressed above raised beneath, pubescent on nerves beneath; lateral veins 6 pairs, convergent forming intra marginal venation; petioles 5-7 mm long, brownish pubescent. Inflorescence axillary and terminal, spike 1-3.5 cm long; peduncle brownish pubescent. Flowers $2.5-4 \mathrm{~mm}$ across, bract 2-3.1 x 1-1.5 mm, ovate, acute at apex, margin ciliate, densely appressed purple-brown pubescent, caducous; bracteoles 2 on either side of the calyx, 2-2.4 x 0.9 - $1.1 \mathrm{~mm}$, more or less similar to bract, lanceolate elliptic, acute at apex, margin ciliate, caduceus. Calyx tube 1.5-1.7 x 1-3 mm, pubescent; lobes 5, 2 x $1 \mathrm{~mm}$ with dark purplish hairs, ovate, obtuse, margin lacerate. Petals 5, 2.5-3 x 1.7-2 mm, white or pale yellow, glabrous, broadly elliptic- oblong with 5 conspicuous nerves, obtuse at apex, entire at margin. Stamens 25, pentadelphous, as long as or shorter than corolla; filaments $1.5-3 \mathrm{~mm}$; anthers $0.4 \times 0.3 \mathrm{~mm}$. Ovary 3-locular with 2 ovules in each locule; style $1.3-2 \times 0.3 \mathrm{~mm}$, shorter than the corolla, stout, glabrous above, stigma flat 
or capitates; disk pentagonous, pubescent. Fruits $0.9-1.8 \times 0.3-0.4 \mathrm{~cm}$, ellipsoid-oblongoid, calyx persistent; mesocarp thick, fleshy; endocarp thin,68 grooved, 1 seeded. Seeds 15 x $4 \mathrm{~mm}$, oblong, yellowish brown.

Phenology: Flowering from December to March; fruiting from March to May.

Habitat and conservation status: Along the margins of the evergreen forest at 2200-2300 m elevation. We have located only three mature trees within an area of about $05 \mathrm{sq} \mathrm{km}$ at Mathikettan Shola National Park. No special measures have so far been taken for its ex-situ conservation.

Distribution and Ecology: Symplocos nicolsonii is found to occur at the Mathikettan shola National Park in Idukki District, Kerala State, South India. The habitat of the new taxon is an evergreen forest and the associated species are Bhesa indica (Bedd.) Ding Hou, Prunus ceylanica (Wight) Miq., Medinilla sabyadrica Sujanapal \& Sasidh., Impatiens viscosa Bedd. etc.

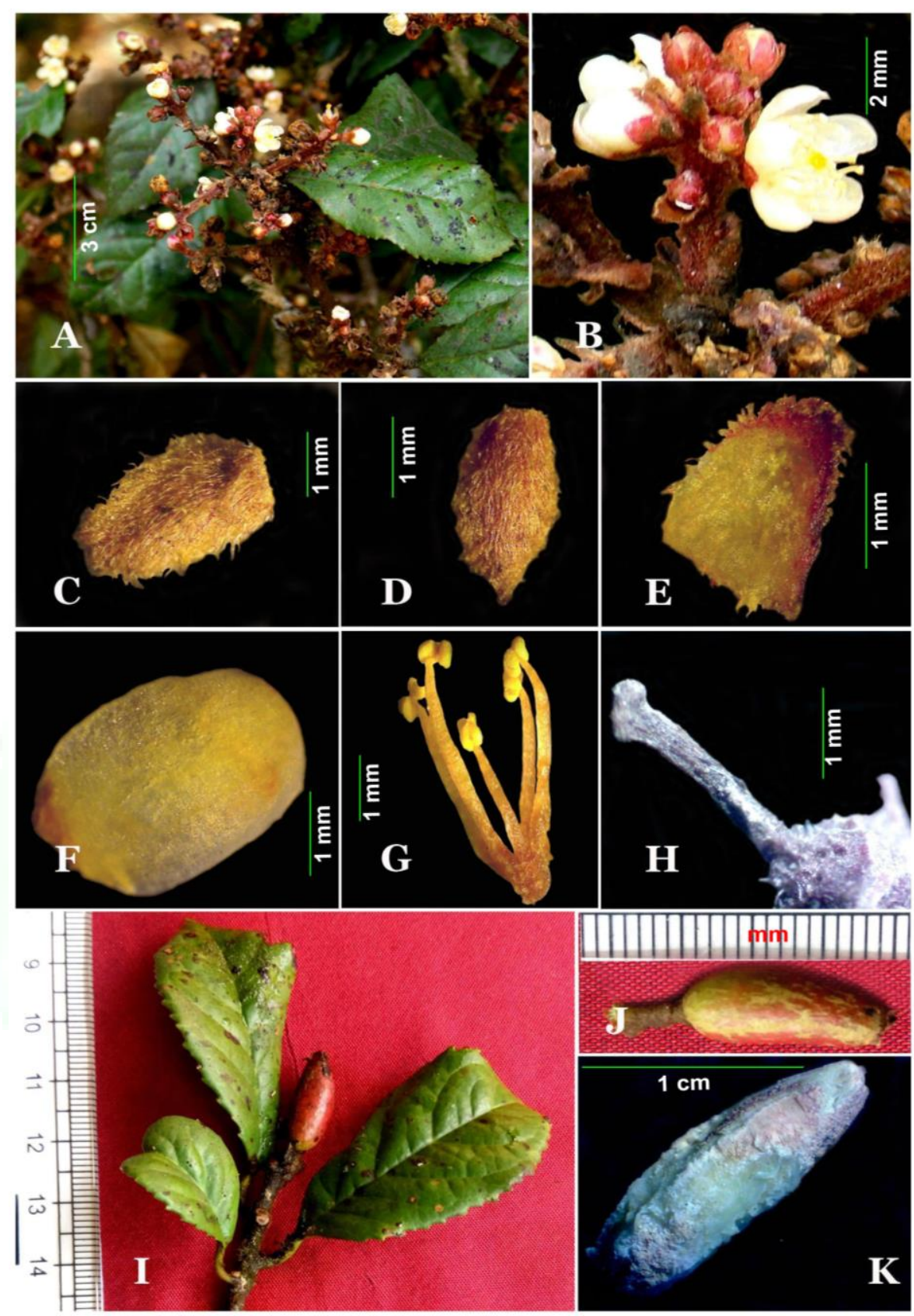

Figure 1: Symplocos nicolsonii R. Jagadeesan, A. Gangaprasad, S. P. Mathew E.S.S. Kumar, et J. Stephan. A. a flowering twig, B. an inflorescence, C. a bract, D. a bracteole, E. a calyx lobe, F. a corolla lobe, G. a bundle of stamen, H. a pistil, I. a fruiting twig, J. a fruit, K. a seed. 
Table 1: Comparison between Symplocos nicolsonii sp. nov. and its allied species S. lancifolia

\begin{tabular}{|c|c|c|}
\hline Characters & $\begin{array}{l}\text { S. nicolsonii sp. } \\
\text { nov. }\end{array}$ & S. lancifolia \\
\hline Petiole & $\begin{array}{l}5-7 \mathrm{~mm} \quad \text { long, } \\
\text { brownish } \\
\text { pubescent }\end{array}$ & $\begin{array}{l}1-3 \mathrm{~mm} \text { long, scarcely } \\
\text { pubescent }\end{array}$ \\
\hline Leaves & $\begin{array}{l}2-5.5 \times 1-3.5 \mathrm{~cm} \text {, } \\
\text { elliptic-obovate, } \\
\text { margin serrate- } \\
\text { revolute, brittle. }\end{array}$ & $\begin{array}{l}7-9 \times 2-3 \mathrm{~cm} \text {, } \\
\text { lanceolate, margin } \\
\text { crenulate-serrulate, } \\
\text { subcoriaceous } \\
\text { membraneous. }\end{array}$ \\
\hline Bract & $\begin{array}{lr}2-3.1 \mathrm{~mm} & \text { long, } \\
\text { ovate, } & \text { densely } \\
\text { appressed } & \\
\text { pubescent } & \end{array}$ & $\begin{array}{l}0.5-2 \mathrm{~mm} \text { long, ovate- } \\
\text { triangular, glabrescent } \\
\text { or glabrous }\end{array}$ \\
\hline Bracteole & $\begin{array}{l}2-2.4 \mathrm{~mm} \text {, } \\
\text { lanceolate-elliptic }\end{array}$ & $0.5-1.5 \mathrm{~mm}$, ovate \\
\hline Calyx tube & $\begin{array}{l}1.5-1.7 \mathrm{~mm} \text {, } \\
\text { pubescent }\end{array}$ & $\begin{array}{l}0.5-1.5 \mathrm{~mm} \text {, glabrous- } \\
\text { puberulous }\end{array}$ \\
\hline Calyx lobe & $\begin{array}{l}2 \times 1 \mathrm{~mm}, \text { ovate, } \\
\text { margin lacerate }\end{array}$ & $\begin{array}{l}1.5 \times 1.5 \mathrm{~mm} \text {, elliptic- } \\
\text { triangular, margin } \\
\text { ciliate }\end{array}$ \\
\hline Stamens & $\begin{array}{l}25 \text {, as long as or } \\
\text { shorter than the } \\
\text { corolla }\end{array}$ & $\begin{array}{l}25-40 \text {, longer than } \\
\text { the corolla }\end{array}$ \\
\hline Disc & Pubescent & Glabrous \\
\hline Style & Hairy at the base & Glabrous \\
\hline Mesocarp & $\begin{array}{l}\text { Thick, fleshy } \\
\text { 6-8groves }\end{array}$ & $\begin{array}{l}\text { Thin } \\
\text { Without groves }\end{array}$ \\
\hline Endocarp & Inconspicuous & (smooth), woody \\
\hline
\end{tabular}

Etymology: Specific epithet of the new taxon is named after late Dr Dan H. Nicolson (1933-2016) who passed away on $2^{\text {nd }}$ June 2016, for his great contributions to the field of botanical nomenclature.

\section{Acknowledgements}

The authors wish to record their sincere thanks to Dr K. N. Gandhi, Harvard University for nomenclatural scrutiny, the Head and Professor, Department of Botany, University of Kerala and the Director, JNTBGRI for the facilities and encouragements.

\section{References}

1. Brand, A. 1901. Symplocaceae. Das Pflanzenreich IV. 242 (Heft 6): 1-100.

2. Fritsch, P. W., Cruz, B.C., Almeda, F., Wang, Y. \& Shi, S. 2006. Phylogeny of Symplocos based on DNA sequences of the chloroplast trnC-trnD intergenic re- gion. Systematic Botany 31: 181-192.

3. Nair, T. S., Rasiya, B. A. and Sibi, M. 2014 Flowering plants of the Western Ghats, India. 1: 1934.

4. Nooteboom, H.P. 1975. Revision of the Symplocaceae of the Old World, New Caledonia excepted. Leiden University Press, Leiden, Netherlands.

5. Stahl, B. 1995. Diversity and distribution of Andean Symplocaceae. pp 397-405. In: S. P. Churchill, H. Balslev, E. Forero \& J.L. Luteyn (eds.), Biodiversity and conservation of neotropical montane forests. The New York Botanical Garden, Bronx, New York.

\section{Cite this article as:}

Jagadeesan R., Gangaprasad A., Sam P. Mathew, Santhosh Kumar E. S., J. Stephan. Symplocos nicolsonii, (symplocaceae) a new species from the Southern Western Ghats, India. Annals of Plant Sciences 5.7 (2016): 1389-1391.

DOI:http://dx.doi.org/10.21746/aps.2016.07.004

Source of support: Nil

Conflict of interest: None Declared 